\title{
Descripción Anatómica del Músculo Extensor del I y II Dedo de la Mano del Zorro Perruno (Cerdocyon thous Linnaeus, 1766)
}

\author{
Anatomical Description of Crab-Eating Fox' (Cerdocyon thous \\ Linnaeus, 1766) Extensor of Digit I and II Muscle
}

Juan Fernando Vélez García*; Juan Sebastián Echeverry Pérez ${ }^{* * *}$ \& Carlos Arturo Sánchez Buitrago***

VÉLEZ, G. J. F.; ECHEVERRY, P. J. S. \& SÁNCHEZ, B, C. A. Descripción anatómica del músculo extensor del I y II dedo de la mano del zorro perruno (Cerdocyon thous Linnaeus, 1766). Int. J. Morphol., 33(4):1455-1459, 2015.

RESUMEN: El zorro perruno (Cerdocyon thous) es el cánido silvestre más común en América del Sur, con una relativa estabilidad poblacional, pero susceptible de ser atropellado y caer en trampas, pudiendo sufrir traumas en sus miembros torácicos, por lo tanto se requiere un conocimiento anatómico específico de sus músculos para intervenciones médicas y quirúrgicas. El objetivo principal de esta investigación fue realizar una descripción anatómica del músculo extensor del I y II dedo del C thous, en la cual, se reporta forma, origen, inserción, inervación e irrigación. Se diseccionaron de superficial a profundo los dos antebrazos de seis especímenes muertos donados por CORPOCALDAS a la Universidad de Caldas. El músculo extensor del I y II dedo en este estudio presentó características anatómicas similares a las reportadas a otras especies pero con una distribución tendinosa variante formando desde tres a cuatro ramas que se distribuyen del I al III dedo, incluso faltando a veces para el III dedo, pero de igual forma presenta una distribución que debe ser conocida para procedimientos que requieran de su conocimiento.

PALABRAS CLAVE: Anatomía; Antebraquial; Canidae; Miología.

\section{INTRODUCCIÓN}

El zorro perruno (Cerdocyon thous) es el miembro de la familia Canidae más común en América del Sur, y tiene una relativa estabilidad poblacional, por lo que es considerado por la IUCN (International Union Conservation Nature) como una especie de preocupación menor (Courtenay \& Maffei, 2008), pero es un cánido que hace parte de la fauna silvestre, la cual se ve afectada por el proceso de colonización de nuevas tierras, reduciéndose cada vez más los espacios naturales necesarios para su supervivencia (Negret, 1984), y en general suele ser perseguida por el humano para el tráfico ilegal, siendo susceptibles de ser vendidos como mascotas, lo que aumenta considerablemente las probabilidades de patologías propias de la domesticación de un animal silvestre y de los posibles accesos violentos por causa de un mal comportamiento de sus captores y cuidadores (Negret; Ojasti \& Dallmeier, 2000), además de los continuos accidentes que pueden sufrir, entre ellos el atrapamiento en cercas y trampas, o la más común, atropellamiento con vehículos a nivel de carreteras (Santiago \&
Oliveira, 2001), eventos en los cuales, los miembros torácicos pueden verse afectados, por lo tanto, se requiere conocer su anatomía.

Los cánidos en general poseen adaptaciones para correr, entre ellas, el hecho de poseer el radio y la ulna articulados de forma que previene rotaciones inapropiadas del antebrazo durante la carrera, además poseen una caracterización bien definida para desplazarse de forma digitígrada (Pessutti, 2001), y para ello, el C. thous presenta unos miembros locomotores cortos y robustos que facilitan su desplazamiento a través de áreas boscosas (Berta, 1982), por lo tanto, su región antebraquial debe contar con una musculatura homologa al perro doméstico (Canis familiaris), como los músculos cráneo-laterales, los cuales permiten la extensión de carpo y dedos (Budras et al., 2007; Dyce et al., 2012; König \& Liebich, 2011), y entre ellos se encuentra un músculo como el extensor del I y II dedo, que varía regularmente en la distribución de los tendones en el perro doméstico

* Departamento de Sanidad Animal, Facultad de Medicina Veterinaria y Zootecnia, Universidad del Tolima, Bogotá, Colombia.

** Programa de Medicina Veterinaria y Zootecnia, Semillero de Investigación en Anatomía Veterinaria, Universidad de Caldas, Manizales, Colombia.

*** Departamento de Salud Animal, Facultad de Ciencias Agropecuarias. Universidad de Caldas en convenio con CORPOCALDAS, Manizales, Colombia. 
(Evans \& de Lahunta, 2013), y aunque se encuentra un estudio de los músculos del antebrazo del $C$. thous (Vaz et al., 2011), éste no se reporta, por lo tanto, con el presente estudio se pretende dar una descripción anatómica que pueda servir como base para procedimientos que involucren conocer la anatomía y variabilidad en la distribución de los tendones de este músculo.

\section{MATERIAL Y MÉTODO}

Se utilizaron seis especímenes que murieron bajo la jurisdicción de CORPOCALDAS (Autoridad ambiental del Departamento de Caldas en Colombia), los cuales, gracias al convenio interadministrativo No. 172-2011, fueron donados y transportados al laboratorio de morfología veterinaria de la Universidad de Caldas, para ser estudiados con su respectivo permiso ambiental. Se fijaron con una solución en mezcla de formol al $10 \%$, aceite mineral al $5 \%$ y ácido fénico al $1 \%$, mediante aplicación en arteria carótida común, reforzando con infiltraciones subcutáneas e intramusculares, y dispuestos por inmersión en la misma solución sin aceite mineral por un período mínimo de 48 horas. Posteriormente a sus miembros torácicos se les realizó disección de superficial a profundo, haciendo énfasis en la parte cráneo-lateral de la región antebraquial (Regio antebrachii) para encontrar el músculo extensor del I y II dedo, y también en el dorso de la mano para observar la distribución de sus tendones. Se revisó este músculo en los doce miembros torácicos y se describió su forma, origen, inserción, inervación e irrigación, llevando registros fotográficos, y describiendo de acuerdo a la terminología de la Nomina Anatomica Veterinaria (International Committee on Veterinary Gross Anatomical Nomenclature, 2012).

\section{RESULTADOS}

El músculo extensor del I y II dedo del C. thous es un músculo que se encuentra profundo en la musculatura cráneolateral del antebrazo, es unipennado, alargado y delgado, con un origen tendino-carnoso que se extiende en los dos tercios distales del margen lateral de la ulna, en el epimisio del $\mathrm{m}$. abductor largo del I dedo, y en el ligamento interóseo; su vientre muscular se extiende hasta el retináculo extensor, y presenta un tendón que pasa profundo al tendón extensor digital común por el retináculo extensor (Fig. 1), y una vez lo atraviesa se ramifica en tendones que se distribuyen de forma variable entre especímenes. En dos especímenes emitió una rama que se dirige medialmente hacia el I dedo formando una aponeurosis que recubre al I metacarpiano, insertándose en un cartílago sesamoideo que se encuentra dorsal a la articulación metacarpofalángica, continuándose como un tendón hasta el proceso extensor de la falange distal del I dedo; y dos ramas para el II dedo, donde una rama se inserta en la cápsula articular metacarpo-falángica a nivel abaxial, y otra rama en la cápsula articular metacarpo-falángica en la cara dorso-axial, donde se une al tendón del m. extensor digital común (Fig. 2A). En tres especímenes se encontró que emite otra rama hacia el III dedo a nivel abaxial que se inserta en la cápsula articular metacarpofalángica y se une al tendón extensor digital común a nivel dorsal de la articulación metacarpo-falángica (Fig. 2B). En un espécimen no se hallaron tendones distribuidos hacia los dedos, sino formando una aponeurosis que se dirige para cubrir el primer dedo, y adhiriéndose en la parte medial del tendón del m. extensor digital común (Fig. 2C). En todos los especímenes se encontró inervado por el ramo profundo del nervio radial una vez atravesó al m. supinador; y se encontró irrigado por la arteria interósea craneal (Fig. 1) y ramas de la arteria interósea caudal que atravesaban el ligamento interóseo.

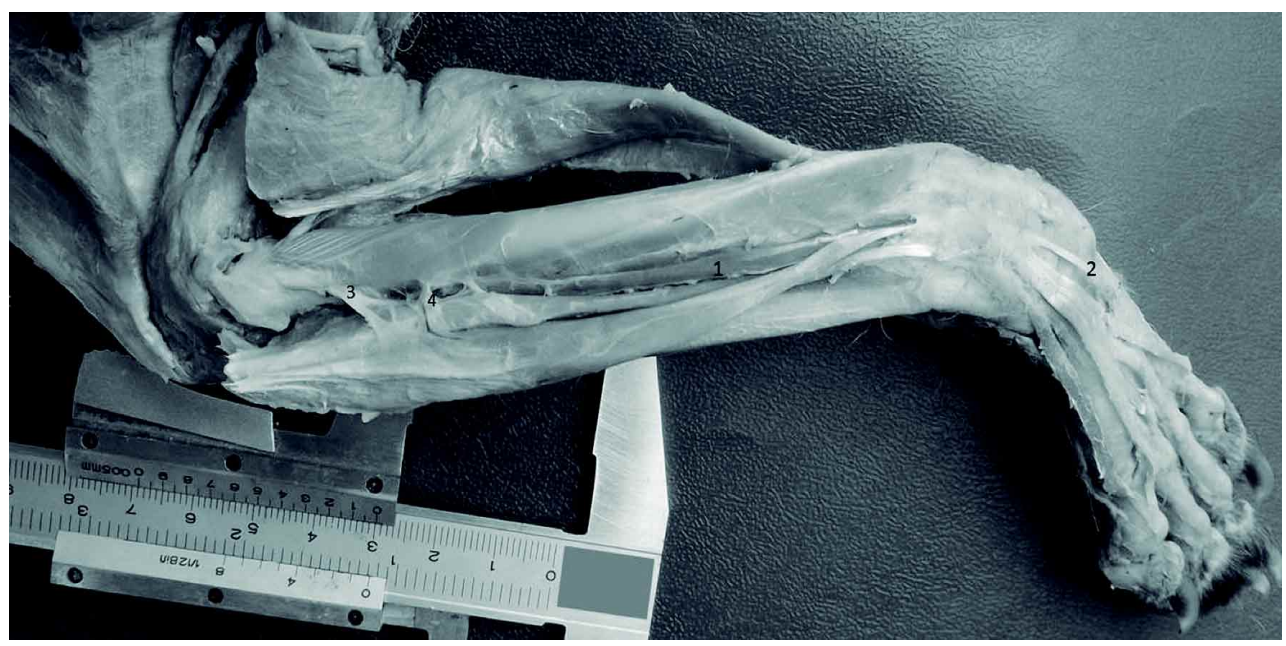

Fig. 1. Disección profunda de la parte cráneo-lateral del antebrazo. Músculo extensor del I y II dedo (1), tendón (2), Ramo profundo del nervio radial (3) y Arteria interósea craneal (4). 


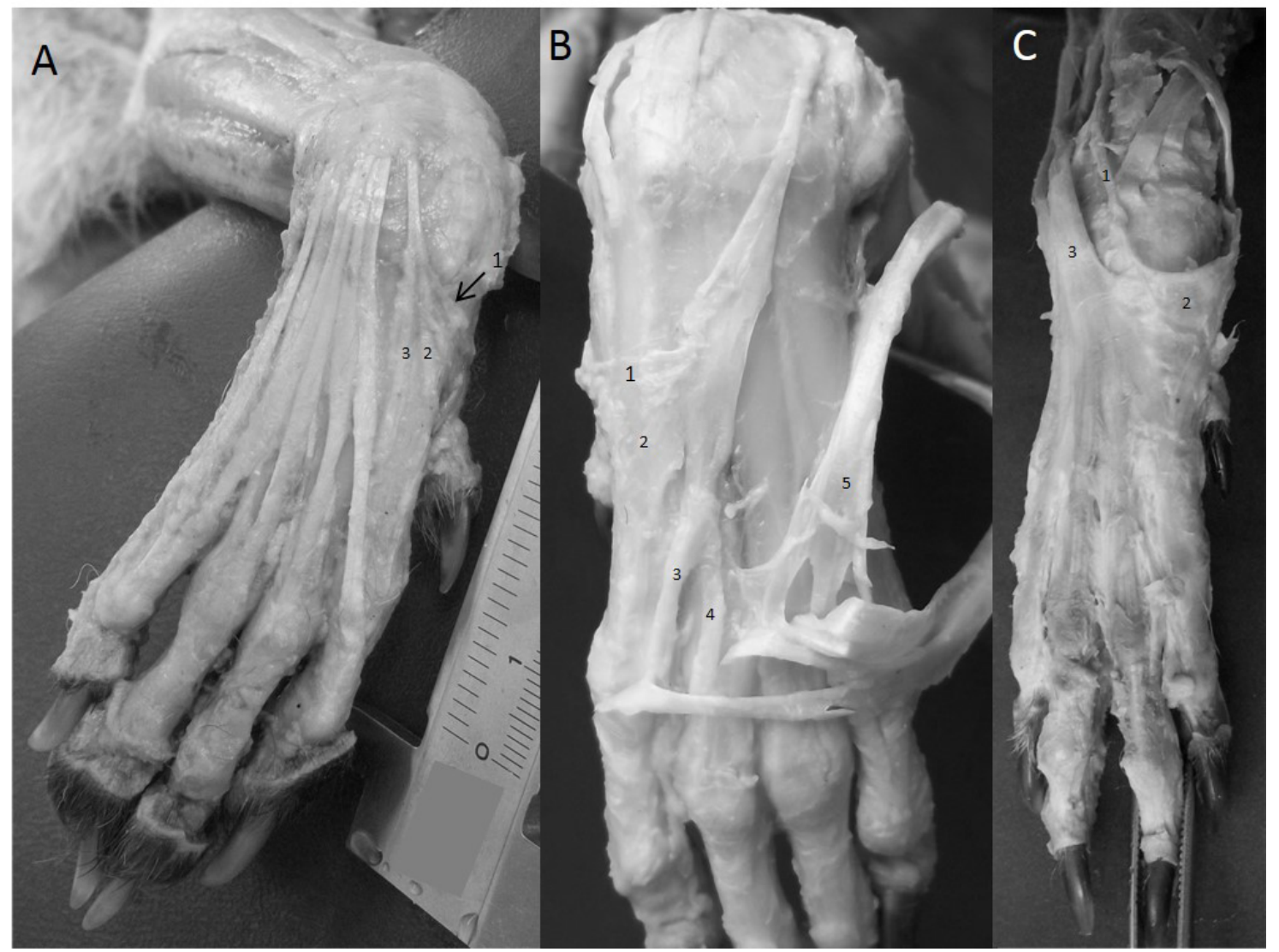

Fig. 2. Ramificación del tendón del m. extensor del I y II. A) Rama para el I dedo (1), rama abaxial para el II dedo (2), rama axial para el II dedo (3); B) rama para el III dedo (4), tendones del m. extensor digital lateral (5); C) El tendón del extensor del I y II dedo (1) forma una aponeurosis (2) que se adhiere al tendón del extensor digital común (3).

\section{DISCUSIÓN}

Este músculo ha sido denominado en el perro doméstico como extensor del I y II dedo debido a que su tendón envía dos ramas, una para el I dedo y otra para la parte axial del II dedo (Budras et al.; Evans \& de Lahunta; Getty, 1982; König \& Liebich), pero en la nomenclatura de la Nomina Anatomica Veterinaria aparecen como músculos independientes (M. extensor digiti I, y M. extensor digiti II), siendo una característica que se presenta de manera ocasional en el perro (Evans \& de Lahunta), y se puede encontrar en Hyaena hyaena donde reportan por separado el m. extensor del II dedo (Spoor \& Badoux, 1986), pero esta separación no fue hallada por nosotros en el C thous, por lo tanto conservamos el nombre de extensor del I y II dedo, aunque encontramos una rama para el III dedo en mayor proporción, lo que nos lleva a sugerir que una vez se apoye este estudio con más especímenes en investigaciones posteriores, se podría denominar mejor como músculo extensor digital medial, ya que no iría sólo para el I y II dedo, y apar- te de esto es un apoyo tendinoso profundo al extensor digital común, siendo el colateral del m. extensor digital lateral, donde este último distribuye tendones profundamente en dedos laterales (Fig. 2B). El extensor del I y II dedo no es reportado en descripciones de los músculos del antebrazo de algunos carnívoros silvestres, como es el caso del Procyon cancrivorus (Lima et al., 2010; Santos et al., 2010a), Nasua nasua (Santos et al., 2010b), Atelocynus microtis, e inclusive en un espécimen de $C$. thous (Vaz et al.), donde en esta última especie corroboramos su presencia en los dos antebrazos de los seis especímenes diseccionados.

El músculo extensor del I y II dedo del C. thous presenta origen en el epimisio del $\mathrm{m}$. abductor largo del I dedo, el cual no se reporta en descripciones de este músculo en el perro y el gato doméstico (Felis catus) (Barone, 2000; Budras et al.; Evans \& de Lahunta; Getty; König \& Liebich); y Barone y Budras et al. reportan su origen en el ligamento 
interóseo como sucede en el C. thous. Estos orígenes no se nombran en otros miembros del Orden Carnivora, como Puma concolor (Concha et al., 2004), Taxidea taxus (Moore et al., 2013), y H. hyaena (Spoor \& Badoux). La distribución de sus tendones varía de acuerdo a lo reportado para el perro doméstico, donde sólo se reportan dos ramas que van para el I y II dedo (Barone; Budras et al.; Evans \& de Lahunta; Getty; König \& Liebich), difiriendo de lo hallado en el $C$. thous, donde se emiten desde tres a cuatro ramas que se dirigen entre el I y III dedo, o incluso formando una aponeurosis que se adhiere al tendón del m. extensor digital común apoyando su función directamente, sin emitir ramas tendinosas individuales para los dedos. La formación de una rama para el III dedo así como se encontró en el C. thous se puede hallar de manera ocasional en el perro doméstico (Evans \& de Lahunta; Getty). Por otro lado, en el gato doméstico se reporta la formación de tres ramas, donde una se dirige para el I dedo y las otras dos para el II dedo (König \& Liebich), similar a lo que puede suceder en $C$. thous, aunque Barone en el gato doméstico lo reporta similar al patrón común del perro doméstico. La inserción de estos tendones en el C. thous es similar a la reportada en el perro doméstico (Barone; Budras et al.; Evans \& de Lahunta), aunque en el C. thous encontramos fijaciones en las cápsulas articulares metacarpo-falángicas que deben contribuir a su estabilización. En carnívoros silvestres como P. concolor (Concha et al.) se describe insertado en las falanges medias del
I y II dedo; en T. taxus (Moore et al.) en las falanges proximales del I y II dedo; y en H. hyaena (Spoor \& Badoux) se describe una unión tendinosa con el extensor digital común para el II dedo únicamente, lo que hace diferenciar estas especies del C. thous. La inervación es la misma que se describe en el perro doméstico (Barone; Budras et al.; Evans \& de Lahunta), al igual que la irrigación reportada por Evans \& de Lahunta, aunque difiere con lo reportado por Barone y Budras et al., quienes no mencionan la arteria interósea caudal, como si se encontró en el C. thous. La presencia de un cartílago sesamoideo en el dorso de la articulación metacarpo-falángica del I dedo se encontró en todos los especímenes de $C$. thous, y no ha sido reportado en el perro doméstico por Barone, Budras et al., Evans \& de Lahunta, Getty y König \& Liebich.

En conclusión el músculo extensor del I y II dedo del C. thous presenta una disposición anatómica adecuada para apoyar la función de los otros músculos extensores digitales, la cual es necesaria para una locomoción en cuadripedestación digitígrada, y de acuerdo a la distribución de sus tendones nos lleva a sugerir que permite la extensión y aducción del primer dedo, y además apoya la función extensora del m. extensor digital común en el II y/ o III dedo, y aunque es una especie del Orden Carnivora presenta diferencias inter e intraespecíficas que deberían ser tenidas en cuenta en procedimientos médicos y quirúrgicos que requieran de su conocimiento.

VÉLEZ, G. J. F.; ECHEVERRY, P. J. S.; SÁNCHEZ, B, C. A. Anatomical description of crab-eating fox' (Cerdocyon thous linnaeus, 1766) extensor of digit I and II muscle. Int. J. Morphol., 33(4):1455-1459, 2015.

SUMMARY: The crab-eating fox (Cerdocyon thous) is the most common wild canid in South America. With a relative demographic stability, this animal is susceptible of being hit and falling into traps, and can suffer trauma in its thoracic limbs; therefore a specific anatomical knowledge about its muscles is necessary for medical and surgical procedures. The main aim of this research was to describe the anatomy of the extensor muscle of digit I and II of the C. thous reporting form, origin, insertion, innervation and irrigation. The forearms of six donated dead specimens from CORPOCALDAS to Caldas University were dissected from superficial to deep. In this research, the extensor muscle of digit I and II showed similar anatomical features to those reported for other species but with a variant tendinous distribution that forms from three to four branches distributed from digit I to III. Although sometimes this distribution does not exist for the digit III; this similar distribution must be reported for procedures which require this knowledge.

KEY WORDS: Anatomy; Antebrachial; Canidae; Myology.

\section{REFERENCIAS BIBLIOGRÁFICAS}

Barone, R. Anatomie comparée des mammifères domestiques. Tome 2, Arthrologie et Myologie. Paris, Vigot, 2000.

Budras, K. D.; McCarthy, P. H.; Fricke, W. \& Richter, R. Anatomy of the Dog. 5th ed. Frankfurt, Schlütersche, 2007.

Berta, A. Cerdocyon thous. Mamm. Species, 186:1-4, 1982.
Concha, I.; Adaro, L.; Borroni, C. \& Altamirano, C. Anatomical considerations over the intrinsic musculature of thoracic limb of puma (Puma concolor). Int. J. Morphol., 22(2):121-5, 2004.

Courtenay, O. \& Maffei, L. Cerdocyon thous. The IUCN Red List of Threatened Species, 2008:e.T4248A10701650, 2008. Disponible en: http://dx.doi.org/10.2305/IUCN.UK.2008.RLTS. T4248A10701650.en 
Dyce, K. M.; Sack, W. O. \& Wensing, C. J. G. Anatomía veterinaria. $4^{\text {a }}$ ed. México D. F., El Manual Moderno, 2012.

Evans, H. E. \& de Lahunta, A. Miller's Anatomy of the Dog. 4th ed. St. Louis, Elsevier Saunders, 2013.

Getty, R. Sisson y Grossman: Anatomía de los animales domésticos. Tomo II. $5^{\text {a }}$ ed. Bogotá, Masson, 1982.

International Committee on Veterinary Gross Anatomical Nomenclature (ICVGAN). Nomina Anatomica Veterinaria. 5a rev. ed. Knoxville, Editorial Committee Hannover, Columbia, Gent, Sapporo, 2012.

König, H. E. \& Liebich, H. G. Anatomía de los animales domésticos. Tomo I: Aparato locomotor. $2^{\mathrm{a}}$ ed. Madrid, Médica Panamericana, 2011.

Lima, V. M.; Pereira, F. C. \& Pereira, K. F. Estudo morfológico dos músculos do antebraço de mão-pelada (Procyon cancrivorus Cuvier, 1798). Biosci. J., 26(1):109-14, 2010.

Moore, A. L.; Budny, J. E.; Russell, A. P. \& Butcher, M. T. Architectural specialization of the intrinsic thoracic limb musculature of the American badger (Taxidea taxus). J. Morphol., 274(1):35-48, 2013.

Negret, R. Ecología y manejo de Fauna Silvestre. Austin, Universidad de Texas, Departamento Administrativo de Intendencias y Comisarías, 1984.

Ojasti, J. \& Dallmeier, F. Manejo de Fauna Silvestre Neotropical. Washington D.C., SI/MAB Series No. 5 Smithsonian Institution/ MAB Program, 2000.

Pessutti, C.; Santiago, M. E. B. \& Oliveira, L. T. F. Order Carnivora, Family Canidae (Dogs, Foxes, Maned Wolves): Biology. En: Fowler, M. E. \& Cubas, Z. S. (Eds.). Biology, Medicine and Surgery of South American Wild Animals. Ames, Iowa State University Press, 2001. pp.279-85.

Santiago, M. E. B. \& Oliveira, L. T. F. Order Carnivora, Family Canidae (Dogs, Foxes, Maned Wolves): Medicine. En: Fowler, M. E. \& Cubas, Z. S. (Eds.). Biology, Medicine and Surgery of South American Wild Animals. Ames, Iowa State University Press, 2001. pp.285-90.

Santos, A. C.; Machado Bertassoli, B.; Rosa, R. A.; Carvalho, A. F. \& Furlanetto Mançanares, C. A. Miologia comparada do membro torácico do mão-pelada (Procyon cancrivorus, G. Cuvier, 1798). Rev. FZVA, 17(2):262-75, 2010 a.

Santos, A. C. D.; Machado Bertassoli, B.; Oliveira, V. C.; Carvalho, A. F.; Rosa, R. A. \& Furlanetto Mançanares, C. A. Morfologia dos músculos do ombro, braço e antebraço do quati (Nasua nasua Linnaeus, 1758). Biotemas, 23(3):16773, 2010b.
Spoor, C. F. \& Badoux, D. M. Descriptive and functional morphology of the neck and forelimb of the striped hyena (Hyaena hyaena, L. 1758). Anat. Anz., 161(5):375-87, 1986.

Vaz, M. G. R.; de Lima, A. R.; de Souza, A. C. B.; Pereira, L. C. \& Branco, É. Estudo morfológico dos músculos do antebraço de cachorro-do-mato-de-orelhas-curtas (Atelocynus microtis) e cachorro-do-mato (Cerdocyon thous). Biotemas, 24(4):121-7, 2011.

Dirección para Correspondencia:
Juan Fernando Vélez García
Departamento de Salud Animal
Facultad de Medicina Veterninaria y Zootecnia
Universidad de Tolima
Ibagué
COLOMBIA

Email:jfvelezg@ut.edu.co

Recibido: 23-12-2014

Aceptado: 02-09-2015 